\title{
Second-order conditioning in Drosophila
}

\author{
Christopher J. Tabone and J. Steven de Belle ${ }^{1,2}$ \\ School of Life Sciences, University of Nevada, Las Vegas, Las Vegas, Nevada 89154, USA
}

\begin{abstract}
Associative conditioning in Drosophila melanogaster has been well documented for several decades. However, most studies report only simple associations of conditioned stimuli (CS, e.g., odor) with unconditioned stimuli (US, e.g., electric shock) to measure learning or establish memory. Here we describe a straightforward second-order conditioning (SOC) protocol that further demonstrates the flexibility of fly behavior. In SOC, a previously conditioned stimulus (CSI) is used as reinforcement for a second conditioned stimulus (CS2) in associative learning. This higher-order context presents an opportunity for reassessing the roles of known learning and memory genes and neuronal networks in a new behavioral paradigm.
\end{abstract}

Second-order conditioning is a form of higher-order associative learning wherein a previously conditioned stimulus (CS1) can associate with a second conditioned stimulus (CS2) to elicit a conditioned response. Initial training involves pairing of CS1 with an unconditioned stimulus (US) during a first-order conditioning (FOC) session followed by a second-order conditioning (SOC) session in which CS1 is paired subsequently with a novel stimulus, CS2. If SOC is successful, an animal will demonstrate a conditioned response to CS2 similar to CS1, even though it has not been exposed to the original US during CS2+CS1 association. Although SOC was originally described by Pavlov (1927) and has been thoroughly studied by psychologists for nearly four decades (Rizley and Rescorla 1972), modern neuroscience has only recently turned to this paradigm as a means for understanding the mechanisms of learning and memory (Gewirtz and Davis 2000). SOC presents a unique opportunity to investigate the internal transfer of information from one conditioned stimulus to another (CS1 $\rightarrow$ CS2), leading to a conditioned response.

The ability to form higher-order associations is prevalent in nature. SOC studies have spanned numerous animal models, including sea slugs (Hawkins et al. 1998), honeybees (Takeda 1961; Grossman 1971; Bitterman et al. 1983; Hussaini et al. 2007), crickets (Mizunami et al. 2009), pigeons (Rashotte et al. 1977; Rescorla 1979), rats (Rizley and Rescorla 1972; Holland and Rescorla 1975; Debiec et al. 2006), and humans (Jara et al. 2006; Karazinov and Boakes 2007). While investigations of learning with flies have demonstrated complex behavior such as sensory preconditioning, the results of previous vision-based SOC studies were only significant when results from two different experiments were pooled together (Brembs and Heisenberg 2001). This finding encouraged our investigation of SOC in Drosophila melanogaster in which we used a robust olfactory-based conditioning paradigm. Flies have served as a model for many types of learning and memory studies (Pitman et al. 2009) and are arguably the best understood animal genetics model as well. Their ease of manipulation using transgenic technologies and conditional expression of neuron-silencing transgenes (Keene and Waddell 2007), along with the availability of numerous learning- and memory-associated mutations (McGuire et al. 2005) makes the fly an extremely powerful system for examining neural mechanisms of behavioral plasticity.

\footnotetext{
1Present address: Dart Neuroscience, San Diego, CA 92121, USA. ${ }^{2}$ Corresponding author.

E-mail sdebelle@dartneuroscience.com.

Article is online at http://www.learnmem.org/cgi/doi/10.1101//m.2035411.
}

We used the Pavlovian olfactory conditioning T-maze paradigm (Tully and Quinn 1985) for all experiments. Flies were Canton special raised at $24 \pm 0.5^{\circ} \mathrm{C}$ and $35 \% \pm 5 \%$ humidity on standard Bloomington Drosophila medium (http://flystocks.bio. indiana.edu/Fly_Work/media-recipes/bloomfood.htm) with a 12:12-h light:dark cycle. Experiments were performed at $88 \% \pm$ $5 \%$ humidity and $24^{\circ} \pm 0.5^{\circ} \mathrm{C}$ under dim red light. For training, groups of approximately 1002 - to 5 -d-old adults were aspirated into an acrylic tube lined with an embedded double-wound copper coil to deliver electric foot shock ( $90 \mathrm{~V}$ dc; US). Current was provided by a dc-regulated power supply (Circuit Specialists). Three odorants, benzaldehyde (BEN; Aldrich), 4-methylcyclohexanol (MCH; Aldrich), and 3-octanol (OCT; Aldrich) were suspended in heavy mineral oil (Mallinckrodt) at dilutions of $8 \times 10^{-4}, 1.4 \times 10^{-3}$, and $2 \times 10^{-3}$, respectively. Room air was bubbled through the odorants in a custom-built automated delivery system (Analytical Research Systems) (Fig. 1A) at a flow rate of $650 \mathrm{~mL} \mathrm{~min}{ }^{-1}$. Presentation of all stimuli was controlled by FieldPoint relays using LabVIEW software (National Instruments).

Our assessment of SOC in Drosophila used three different protocols involving alternated pairing and unpairing of conditioned stimuli (Fig. 1B), adapted from earlier studies by Rizley and Rescorla (1972) and Takeda (1961). The paired-paired (P-P) protocol was designed with both CS1 and CS2 temporally paired with the US and CS1, respectively. The paired-unpaired (P-U) protocol retained pairing of CS1 and the US but delayed association of CS2 with CS1 by $45 \mathrm{sec}$. Alternatively, the unpaired-paired (U-P) protocol delayed association of CS1 with the US by $45 \mathrm{sec}$, while CS2 and CS1 remained paired. After training, flies were transferred to a choice point between converging air currents and tested for their responses to either first- or second-order conditioned stimuli. A performance index (PI) was calculated for each test as the normalized percent of flies that avoided a previously conditioned stimulus (CS1 or CS2, depending on the protocol) vs. an unreinforced stimulus $\left(\mathrm{CS}^{-}\right)$. A PI of 100 indicates $100 \%$ avoidance of a previously conditioned stimulus, while a PI of 0 represents a 50:50 distribution (i.e., no learning). We calculated a full PI as the average of two tests using separate groups of flies. CS1 (FOC) or CS2 (SOC) and $\mathrm{CS}^{-}$odors in the first test were reversed in the second. In this way we accounted for a possible odor bias among different populations of flies (Tully and Quinn 1985).

In our study, three odorants (BEN, MCH, and OCT), deployed in three different protocols $(\mathrm{P}-\mathrm{P}, \mathrm{P}-\mathrm{U}, \mathrm{U}-\mathrm{P})$ with reciprocal tests to account for odor bias, and tests for both FOC and SOC give a total of 36 possible unique experimental combinations. For 
A

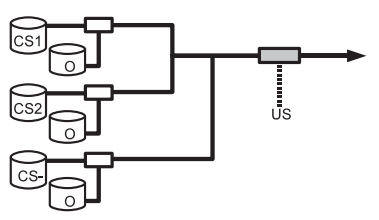

B
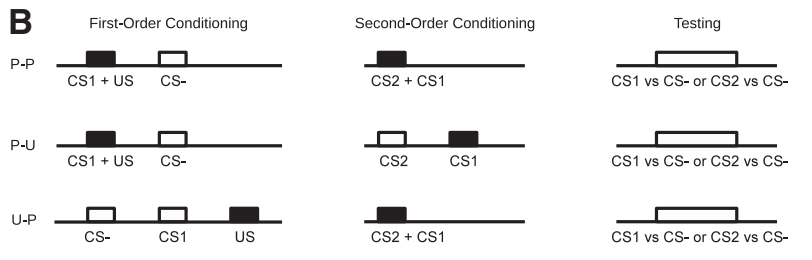

C

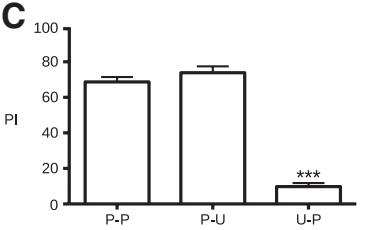

D

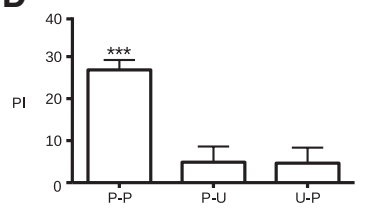

Figure 1. First- and second-order conditioning. (A) Schematic representation of our automated odor- and shock-delivery system. Bubblers contained odorants suspended in mineral oil [BEN $\left(8 \times 10^{-4}\right), \mathrm{MCH}(1.4 \times$ $\left.10^{-3}\right)$, and OCT $\left(2 \times 10^{-3}\right) ; C S 1, C S 2$, and $\mathrm{CS}^{-}$] or mineral oil alone (O) and drew ambient room air using an in-house vacuum system $\left(650 \mathrm{~mL} \mathrm{~min}^{-1}\right)$. Solenoids (Analytical Research Systems; white rectangles) directed airflow by opening or closing in response to computercontrolled relays. Air flowed through teflon-coated tubing (Tygon SE-200; solid lines; arrow indicates direction) from bubblers to solenoids, then into acrylic copper-coil-lined training tubes (gray rectangle). We presented mixed odors by opening two solenoids simultaneously. Electric shock (90 V dc; dotted line) was delivered from a dc-regulated power supply (Circuit Specialists) directly to the training tubes. (B) Timeline representations of training and testing. Squares represent stimuli-solid indicate reinforcement, open indicate the absence of reinforcement. All flies received both FOC and SOC and were tested for their responses to either CS1 $(C)$ or CS2 $(D)$ vs. the $\mathrm{CS}^{-}$. In the paired-paired protocol $(P-P)$, both CS1 and CS2 were reinforced. In the paired-unpaired proto$\mathrm{Col}(\mathrm{P}-\mathrm{U}), \mathrm{CS} 2$ preceded CS1 by $45 \mathrm{sec}$, while in the unpaired-paired protocol (U-P), CS1 preceded the US by $45 \mathrm{sec}$. During the SOC phase of the $\mathrm{P}-\mathrm{P}$ and $\mathrm{U}-\mathrm{P}$ protocols, CS2 was presented alone for $7 \mathrm{sec}$, followed by simultaneous presentation of both CS1 and CS2. (C) Pairing stimuli during $\mathrm{FOC}$ was required to generate a conditioned response to CS1 vs. the $\mathrm{CS}^{-}$(ANOVA, $F_{(2,21)}=152.0, P<0.0001$; Tukey, $P \leq 0.05$ ). Note that unpairing of stimuli during $S O C$ did not reduce the first-order conditioned responses of flies using the $\mathrm{P}-\mathrm{U}$ protocol. Bars indicate mean \pm SEM; $n=8 /$ bar. (D) Pairing of stimuli during both FOC and SOC was required to generate a conditioned response to CS2 vs. the $\mathrm{CS}^{-}\left(\right.$ANOVA, $F_{(2,21)}=14.68, P<0.0001$; Tukey, $\left.P \leq 0.05\right)$. Bars indicate mean \pm SEM; $n=8 /$ bar.

expedience, we focused on a subset of odor pairings. When measuring FOC responses, $\mathrm{MCH}$ and OCT were exchanged as CS1 and the $\mathrm{CS}^{-}$, while BEN remained the CS2 odor. Similarly, when we measured SOC responses, BEN and MCH alternated as CS2 and the $\mathrm{CS}^{-}$, while OCT remained the CS1 odor. Tests involving alternative combinations of CS1, CS2, and $\mathrm{CS}^{-}$odors yielded results similar to those reported here (data not shown).

In FOC, flies were exposed to a blank odor (air bubbled through mineral oil) for $90 \mathrm{sec}$, followed by a 60 -sec exposure to the CS1 odor paired with 121.25 -sec pulses of 90 - $\mathrm{V}$ dc shock delivered every $5 \mathrm{sec}$. The training tube was flushed for $45 \mathrm{sec}$ with the blank odor followed by a presentation of an unreinforced $\mathrm{CS}^{-}$odor for an additional $60 \mathrm{sec}$. First-order conditioning was repeated three times with 10-min rest intervals of no odor or airflow.

SOC began with exposure to the blank odor for $90 \mathrm{sec}$ followed by presentation of a novel CS2 alone for $7 \mathrm{sec}$. The previously conditioned CS1 was then presented in 124 -sec pulses alternating with a blank odor every $5 \mathrm{sec}$ for $60 \mathrm{sec}$, paired with a constant flow of CS2 odor. Pulsing of the CS1 odor generated stronger second-order conditioned responses than constant exposure (data not shown). Paired odors converged in the airflow before entering the training tube. The tube was then flushed with blank odor for $45 \mathrm{sec}$. $\mathrm{CS}^{-}$was not presented to the flies during the second-order training phase of the experiment to minimize the time between conditioning and testing. Absence of the $\mathrm{CS}^{-}$was shown to have only minimal influence on performance following FOC (Masek and Heisenberg 2008). After repeating second-order conditioning three times with 10-min rest intervals, flies were tested for their avoidance of either first- or second-order conditioned stimuli.

When CS1 and the US were explicitly unpaired during FOC, we observed a significant decline in avoidance of the firstorder conditioned stimulus (ANOVA, $F_{(2,21)}=152.0, P<0.0001$; Tukey, $P \leq 0.05$; Fig. 1C). As previously observed in FOC experiments, inserting a 45 -sec gap between shock and odor presentation severely reduces avoidance of a conditioned odor (Tully and Quinn 1985). Furthermore, the lack of a significant difference between the paired-unpaired $(\mathrm{P}-\mathrm{U})$ and paired-paired $(\mathrm{P}-\mathrm{P})$ groups indicates that unpairing second-order stimuli has no distinguishable effect on FOC.

During SOC tests, flies demonstrated a significant avoidance of second-order stimuli compared to either unpaired first-order or second-order controls (ANOVA, $F_{(2,21)}=14.68, P<0.0001$; Tukey, $P \leq 0.05$; Fig. 1D). Coincident exposure to both first-order $(\mathrm{CS} 1+\mathrm{US})$ and second-order (CS2+CS1) stimuli is required to form a conditioned response to the second-order stimulus. Explicit unpairing during either first- or second-order conditioning with a 45 -sec gap in stimulus presentation was sufficient to disrupt this association.

In our SOC paradigm, both CS1 and CS2 use the same sensory modality and are presented simultaneously during training. Therefore, flies must be able to distinguish both CS1 and CS2 components. Processing of odor mixtures has been previously investigated in Drosophila (Silbering and Galizia 2007) but not in the context of associative conditioning in adults. We conducted a series of four shock-associated FOC experiments to examine the flies' capacity to discriminate components of odor mixtures during both training and testing (Fig. 2A). In our first protocol, flies were conditioned to avoid an odor mixture $(\mathrm{OCT}+\mathrm{BEN})$ and tested for their avoidance of one component of this mixture (OCT). Protocol 2 is the inverse of protocol 1, with one component (OCT) of an unreinforced mixture (OCT+BEN)
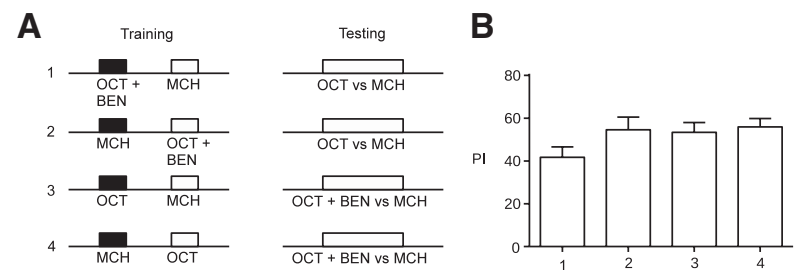

Figure 2. Odor discrimination. (A) Timeline representations of training and testing. Squares represent odor-solid indicate electric shock (90 V dc) reinforcement, open indicate the absence of reinforcement. We assessed discrimination of odors from odor mixtures during both training (protocols 1 and 2) and testing (protocols 3 and 4). (B) Flies demonstrated significant avoidance of all conditioned stimuli ( $t$-test, $t_{(13)}=8.64$, $P<0.0001 ; t_{(13)}=9.13, P<0.0001 ; t_{(13)}=11.50, P<0.0001 ;$ and $\left.t_{(13)}=14.24, P<0.0001\right)$. Differences between groups were not significant (ANOVA, $\left.F_{(3,52)}=1.779, P=0.1626\right)$. Bars indicate mean $\pm S E M$; $n=14 /$ bar. 
presented during the test. In protocol 3, flies were conditioned to avoid a single odor (OCT) and tested for their avoidance of the same odor in a mixture (OCT $+\mathrm{BEN})$. Protocol 4 is the inverse of protocol 3, with an unreinforced odor (OCT) presented in a mixture $(\mathrm{OCT}+\mathrm{BEN})$ during the test. The PI for all four protocols was significantly greater than zero $\left(t\right.$-test, $t_{(13)}=8.64, P<0.0001$; $t_{(13}=9.13, \quad P<0.0001 ; \quad t_{(13)}=11.50, \quad P<0.0001 ;$ and $t_{(13)}=$ 14.24, $P<0.0001$; Fig. 2B). These results clearly demonstrate that flies possess the capacity to discriminate odors from odor compounds and also illustrates why one sensory modality can be used for concurrent presentation of both CS1 and CS2 during SOC. Furthermore, we observed no significant differences among any of the four protocols (ANOVA, $F_{(3,52)}=1.779, P=0.1626$ ), indicating an equivalence of this capacity during both training and testing.

Exposing flies to a previously conditioned stimulus in the absence of a US can lead to extinction during FOC (Tully and Quinn 1985). During SOC, flies were exposed to the CS1 in the presence of CS2 without the original US. This exposure throughout all three cycles of SOC may have led to the decreased salience of CS1 as a reinforcer of CS2. To test for the possibility of such extinction during SOC, we assayed avoidance of CS1 using two training protocols (Fig. 3A). In one, flies received identical training to the $\mathrm{P}-\mathrm{P}$ protocol described earlier and were exposed to CS2 +CS1 during SOC. The second group received identical FOC but was not exposed to CS1 during SOC. We observed no significant difference between the two groups when testing for FOC response ( $t$-test, $t_{(10)}=1.083, P=0.3042$; Fig. 3B), indicating that unreinforced exposure to CS1 did not accelerate the extinction of this stimulus during SOC. A similar result was observed in studies with Apis mellifera, in which the P-P group still demonstrated a first-order response even after numerous spaced SOC trials (Bitterman et al. 1983).

These experiments clearly demonstrate SOC in D. melanogaster using an olfactory conditioning paradigm. Three FOC cycles followed by three SOC cycles are sufficient to produce an avoidance response to CS2. We also confirmed that flies are capable of discriminating odors from odor mixtures during associative conditioning, a necessary prerequisite for our olfactorybased SOC paradigm. Furthermore, we did not observe extinction

A

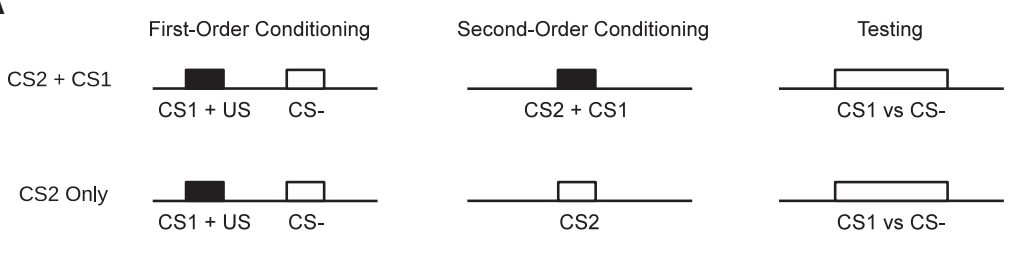

B

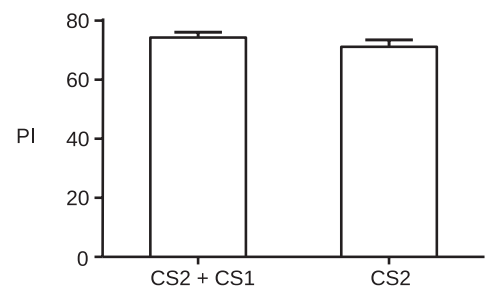

Figure 3. Extinction. (A) Timeline representations of training and testing. Squares represent odor presentation-solid indicate reinforcement, open indicate the absence of reinforcement. We assessed the effect of CS1 extinction during SOC (protocol 1), relative to the control group (protocol 2). (B) Presenting CS1 in the absence of the US did not accelerate extinction of the conditioned response $\left(t\right.$-test, $\left.t_{(10)}=1.083, P=0.3042\right)$. Bars indicate mean $\pm S E M ; n=6 /$ bar. in this study, indicating that our SOC training regime effectively elicits a conditioned response without a significant loss of CS1 salience.

There remain several properties of SOC open for investigation using our protocol. For example, a test of sensory preconditioning would be another, more robust assessment of odor discrimination in the context of our SOC experiment. This would involve presentation of a CS2+CS1 odor mixture prior to pairing of a CS1 component+US reinforcement. Flies would then be tested for their avoidance of the unreinforced CS2 component of the mixture. Although the phenomenon has been demonstrated in Drosophila using a visual-based paradigm (Brembs and Heisenberg 2001), no example of a successful olfactory-based approach yet exists.

Our SOC paradigm may be useful for studying neuronal and molecular mechanisms of extinction in flies (Qin and Dubnau 2010). For example, extinction of CS1 after several rounds of SOC was previously shown to have no effect on the conditioned response to CS2 in rats (Rizley and Rescorla 1972). Combining our robust approach together with Drosophila molecular-genetic tools should lead to a better understanding of the biological processes underlying Rizley and Rescorla's observations on extinction and SOC.

SOC also presents an opportunity to revisit the roles of well-studied learning and memory genes and the neuronal circuits in which they operate. In this regard, we are interested to know whether or not FOC mutants rutabaga (calcium-sensitive adenylyl cyclase) and dunce (cAMP-specific phosphodiesterase) have SOC phenotypes. Drosophila NMDA receptors dNR1 and dNR2 have also been implicated in Pavlovian learning (Xia et al. 2005). Interestingly, blocking NMDA receptor activity in the amygdala prevents second-order fear conditioning in mice (Gewirtz and Davis 1997). Our SOC paradigm will help to determine whether these evolutionarily conserved receptors are required for higher-order learning in flies as well.

Several neurotransmitter-specific circuits necessary for associative learning have been identified in insects. For instance, dopaminergic neuron activity has been shown to provide aversive US reinforcement during FOC in Drosophila (Schwaerzel et al. 2003; Riemensperger et al. 2005), honey bees (Vergoz et al. 2007), and crickets (Unoki et al. 2005). In SOC, a previously conditioned stimulus (CS1), rather than a US, provides reinforcement of CS2. Recent work by Mizunami et al. (2009) has demonstrated that a secondorder conditioned association in crickets can be blocked by administering a dopamine antagonist prior to SOC, indicating that neurotransmission from these neurons is required at this stage of learning. We are currently using a transgenic approach with our SOC paradigm to examine whether dopaminergic neurons function in a similar manner in flies.

While Drosophila behavioral neuroscience has provided some understanding of the mechanisms underlying first-order learning and memory, much less emphasis has been placed on studies of more complex and ecologically relevant behavior. The work presented here demonstrates the fly's capacity for higher-order associative learning and offers a simple and reliable method for investigating the neurobiology of this phenomenon. 


\section{Acknowledgments}

We are grateful to Jeff Anderson, Edward Chan, and Rizwan Jilani for technical assistance and to Xia Wang for comments on a preliminary version of this manuscript. This study was supported by a Summer Session Scholarship (UNLV) to C.J.T, and an NSF grant to J.S.dB (IBN-0237395). Some of this work was conducted by J.S.dB while serving as a Visiting Scientist at the National Science Foundation.

\section{References}

Bitterman ME, Menzel R, Fietz A, Schafer S. 1983. Classical conditioning of proboscis extension in honeybees (Apis mellifera). J Comp Psychol 97: $107-119$.

Brembs B, Heisenberg M. 2001. Conditioning with compound stimuli in Drosophila melanogaster in the flight simulator. J Exp Biol 204: 2849-2859.

Debiec J, Doyère V, Nader K, LeDoux JE. 2006. Directly reactivated, but not indirectly reactivated, memories undergo reconsolidation in the amygdala. Proc Natl Acad Sci 103: 3428-3433.

Gewirtz JC, Davis M. 1997. Second-order fear conditioning prevented by blocking NMDA receptors in amygdala. Nature 388: 471-474.

Gewirtz JC, Davis M. 2000. Using Pavlovian higher-order conditioning paradigms to investigate the neural substrates of emotional learning and memory. Learn Mem 7: 257-266.

Grossmann KE. 1971. Belohnungsverzögerung beim Erlernen einer Farbe an einer künstlichen Futterstelle durch Honigbienen. Z Tierpsychol 29: $28-41$.

Hawkins RD, Greene W, Kandel ER. 1998. Classical conditioning, differential conditioning, and second-order conditioning of the Aplysia gill-withdrawal reflex in a simplified mantle organ preparation. Behav Neurosci 112: 636-645.

Holland PC, Rescorla RA. 1975. Second-order conditioning with food unconditioned stimulus. J Comp Physiol Psychol 88: 459-467.

Hussaini SA, Komischke B, Menzel R, Lachnit H. 2007. Forward and backward second-order Pavlovian conditioning in honeybees. Learn Mem 14: $678-683$.

Jara E, Vila J, Maldonado A. 2006. Second-order conditioning of human causal learning. Learn Motiv 37: 230-246.

Karazinov DM, Boakes RA. 2007. Second-order conditioning in human predictive judgements when there is little time to think. QJ Exp Psychol 60: $448-460$.

Keene AC, Waddell S. 2007. Drosophila olfactory memory: Single genes to complex neural circuits. Nat Rev Neurosci 8: 341-354.
Masek P, Heisenberg M. 2008. Distinct memories of odor intensity and quality in Drosophila. Proc Natl Acad Sci 105: 15985-15990.

McGuire SE, Deshazer M, Davis RL. 2005. Thirty years of olfactory learning and memory research in Drosophila melanogaster. Prog Neurobiol 76: $328-347$.

Mizunami M, Unoki S, Mori Y, Hirashima D, Hatano A, Matsumoto Y. 2009. Roles of octopaminergic and dopaminergic neurons in appetitive and aversive memory recall in an insect. BMC Biol 7: 46.

Pavlov IP. 1927. Conditioned reflexes. Oxford Press, Oxford, UK.

Pitman JL, DasGupta S, Krashes MJ, Leung B, Perrat PN, Waddell S. 2009. There are many ways to train a fly. Fly 3: 3-9.

Qin H, Dubnau J. 2010. Genetic disruptions of Drosophila Pavlovian learning leave extinction learning intact. Genes Brain Behav 9: 203-212.

Rashotte ME, Griffin RW, Sisk CL. 1977. Second-order conditioning of the pigeon's keypeck. Anim Learn Behav 5: 25-38.

Rescorla RA. 1979. Aspects of the reinforcer learned in second-order Pavlovian conditioning. J Exp Psychol Anim B 5: 79-95.

Riemensperger T, Völler T, Stock P, Buchner E, Fiala A. 2005. Punishment prediction by dopaminergic neurons in Drosophila. Curr Biol 15: $1953-1960$.

Rizley RC, Rescorla RA. 1972. Associations in second-order conditioning and sensory preconditioning. J Comp Physiol Psych 81: 1-11.

Schwaerzel M, Monastirioti M, Scholz H, Friggi-Grelin F, Birman S, Heisenberg M. 2003. Dopamine and octopamine differentiate between aversive and appetitive olfactory memories in Drosophila. J Neurosci 23: $10495-10502$.

Silbering AF, Galizia CG. 2007. Processing of odor mixtures in the Drosophila antennal lobe reveals both global inhibition and glomerulus-specific interactions. J Neurosci 27: 11966-11977.

Takeda K. 1961. Classical conditioned response in the honey bee. J Insect Physiol 6: $168-179$.

Tully T, Quinn WG. 1985. Classical conditioning and retention in normal and mutant Drosophila melanogaster. J Comp Physiol A 157: 263-277.

Unoki S, Matsumoto Y, Mizunami M. 2005. Participation of octopaminergic reward system and dopaminergic punishment system in insect olfactory learning revealed by pharmacological study. Eur J Neurosci 22: 1409-1416.

Vergoz V, Roussel E, Sandoz JC, Giurfa M. 2007. Aversive learning in honeybees revealed by the olfactory conditioning of the sting extension reflex. PLoS One 2. e288. doi: 10.1371/ journal.pone.0000288.

Xia S, Miyashita T, Fu TF, Lin WY, Wu CL, Pyzocha L, Lin IR, Saitoe M, Tully T, Chiang AS. 2005. NMDA receptors mediate olfactory learning and memory in Drosophila. Curr Biol 15: 603-615.

Received October 7, 2010; accepted in revised form January 24, 2011. 


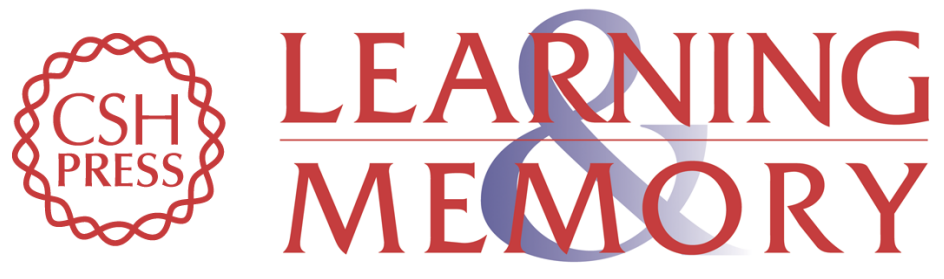

\section{Second-order conditioning in Drosophila}

Christopher J. Tabone and J. Steven de Belle

Learn. Mem. 2011, 18:

Access the most recent version at doi:10.1101//m.2035411

References This article cites 28 articles, 6 of which can be accessed free at: http://learnmem.cshlp.org/content/18/4/250.full.html\#ref-list-1

License

Email Alerting Receive free email alerts when new articles cite this article - sign up in the box at the Service top right corner of the article or click here. 